\title{
Digital Jordan Curve Theorems
}

\author{
Christer O. Kiselman \\ Uppsala University, Department of Mathematics \\ P. O. Box 480, SE-751 06 Uppsala, Sweden \\ kiselman@math.uu.se \\ http://www.math.uu.se/ kiselman
}

\begin{abstract}
Efim Khalimsky's digital Jordan curve theorem states that the complement of a Jordan curve in the digital plane equipped with the Khalimsky topology has exactly two connectivity components. We present a new, short proof of this theorem using induction on the Euclidean length of the curve. We also prove that the theorem holds with another topology on the digital plane but then only for a restricted class of Jordan curves.
\end{abstract}

\section{Introduction}

The classical Jordan curve theorem says that the complement of a Jordan curve in the Euclidean plane $\mathbf{R}^{2}$ consists of exactly two connectivity components. Efim Khalimsky's digital Jordan curve theorem states the same thing for the digital plane $\mathbf{Z}^{2}$. Of course we must use a suitable definition of the concept of digital Jordan curve, as well as a suitable topology on $\mathbf{Z}^{2}$. In this case $\mathbf{Z}^{2}$ is given the Cartesian product topology of two copies of the digital line $\mathbf{Z}$ equipped with the Khalimsky topology.

A proof of Khalimsky's theorem was published in 1990 by Khalimsky, Kopperman and Meyer [1990]. They refer to earlier proofs by Khalimsky (E. D. Halimskiı) $[1970,1977]$. Our first purpose in this note is to present a new, short proof.

The idea of the proof is simple. For the smallest Jordan curves (having four or eight points) the conclusion of the theorem can be proved by inspection. Given any other Jordan curve $J$, we construct a Jordan curve $J^{\prime}$ which has shorter Euclidean length and is such that its complement has as many components as the complement of $J$. Since the possible Euclidean lengths form a discrete set, this procedure will lead to one of the smallest Jordan curves, for which the theorem is already established. The construction of $J^{\prime}$ can intuitively be described as follows: attack $J$ where its curvature is maximal and shorten it there; it cannot offer resistance from within.

We then consider a topology on $\mathbf{Z}^{2}$ which is not a product topology. In contrast to the Khalimsky topology it has the property that every point is either open or closed-there are no mixed points as in the Khalimsky plane. We prove that the Jordan curve theorem holds for this topology for a restricted class of Jordan curves.

G. Borgefors, I. Nyström, and G. Sanniti di Baja (Eds.): DGCI 2000, LNCS 1953, pp. 46-56, 2000. (C) Springer-Verlag Berlin Heidelberg 2000 


\section{Connectedness and Adjacency}

A topological space is said to be connected if it is nonempty and the only sets which are both open and closed are the empty set and the whole space. A subset of a topological space is called connected if it is connected as a topological space with the induced topology. ${ }^{1}$ A connectivity component of a topological space is a connected subset which is maximal with respect to inclusion. A component is always closed. A connected subset which is both open and closed is a component (but not necessarily conversely).

If $X$ and $Y$ are topological spaces and $f: X \rightarrow Y$ a continuous mapping, then the image $f(A)$ of a connected subset $A$ of $X$ is connected. We may apply this result to a situation where we define a topology on a set $Y$ using a mapping $f: X \rightarrow Y$ from a topological space $X$ :

Proposition 1. Let $f: X \rightarrow Y$ be a surjective mapping from a connected topological space $X$ onto a set $Y$. Equip $Y$ with the strongest topology such that $f$ is continuous. Then $Y$ is connected.

In particular we shall use this result with $X=\mathbf{R}$ and $Y=\mathbf{Z}$ to define connected topologies on the digital line $\mathbf{Z}$.

In any topological space we shall denote by $N(x)$ the intersection of all neighborhoods of a point $x$. Spaces such that $N(x)$ is always a neighborhood of $x$ were introduced and studied by Aleksandrov [1937]. Equivalently, they are the spaces where the intersection of any family of open sets is open. In such a space all components are open.

The closure of a subset $A$ of a topological space will be denoted by $\bar{A}$. We note that $x \in \overline{\{y\}}$ if and only if $y \in N(x)$.

A Kolmogorov space (Bourbaki [1961:I: $§ 1:$ Exerc. 2]; also called a $T_{0}$-space) is a topological space such that $x \in N(y)$ and $y \in N(x)$ only if $x=y$. It is quite reasonable to impose this axiom; if $x$ belongs to the closure of $\{y\}$ and vice versa, then $x$ and $y$ are indistinguishable from the point of view of topology. (We should therefore identify them and consider a quotient space.)

The separation axiom $T_{1}$ states that $N(x)=\{x\}$. It is too strong to be of interest for the spaces considered here.

Two points $x$ and $y$ in a topological space $Y$ are said to be adjacent if $x \neq y$ and $\{x, y\}$ is connected. We note that $\{x, y\}$ is connected if and only if either $x \in N(y)$ or $y \in N(x)$. We shall say that two points $x, z$ are second adjacent if $x \neq z ; x$ and $z$ are not adjacent; and there exists a third point $y \in Y$ such that $x$ and $y$ are adjacent and $y$ and $z$ are adjacent.

\section{Topologies on the Digital Line}

It is natural to think of $\mathbf{Z}$ as an approximation of the real line $\mathbf{R}$ and to consider mappings $f: \mathbf{R} \rightarrow \mathbf{Z}$ expressing this idea. We may define $f(x)$ to be the integer

\footnotetext{
${ }^{1}$ According to Bourbaki [1961:I: $\left.§ 11: 1\right]$ the empty space is connected. Here I follow the advice of Adrien Douady (personal communication, June 26, 2000). In the present paper it will not matter whether the empty set is said to be connected or not.
} 
closest to $x$; this is well-defined unless $x$ is a half-integer. So when $x=n+\frac{1}{2}$ we have a choice for each $n$ : shall we define $f\left(n+\frac{1}{2}\right)=n$ or $f\left(n+\frac{1}{2}\right)=n+1$ ? If we choose the first alternative for every $n$, thus putting $\left.\left.f^{-1}(n)=\right] n-\frac{1}{2}, n+\frac{1}{2}\right]$, the topology defined in Proposition 1 is called the right topology on $\mathbf{Z}$; if we choose the second, we obtain the left topology on Z; cf. Bourbaki [1961:I:§1: Exerc. 2]. Khalimsky's topology arises if we always choose an even integer as the best approximant of a half-integer. Then the closed interval $\left[-\frac{1}{2}, \frac{1}{2}\right]$ is mapped to 0 , so $\{0\}$ is closed for the Khalimsky topology, whereas the inverse image of 1 is the open interval $] \frac{1}{2}, \frac{3}{2}[$, so that $\{1\}$ is open.

A Khalimsky interval is an interval $[a, b] \cap \mathbf{Z}$ equipped with the topology induced by the Khalimsky topology on $\mathbf{Z}$. A Khalimsky circle is a quotient space $\mathbf{Z}_{m}=\mathbf{Z} / m \mathbf{Z}$ of the Khalimsky line for some even integer $m \geqslant 4$. (If $m$ is odd, the quotient space receives the chaotic topology, which is not interesting.)

\section{Khalimsky Jordan Curves}

Khalimsky, Kopperman and Meyer [1990:3.1] used the following definitions of path and arc in the Khalimsky plane. We just extend them here to any topological space. We modify slightly their definition of a Jordan curve [1990: 5.1]. A Jordan curve in the Euclidean plane $\mathbf{R}^{2}$ is a homeomorphic image of the circle $\mathbf{R} / \mathbf{Z}$, and similarly a Khalimsky Jordan curve is a homeomorphic image of a Khalimsky circle.

Definition 1. Let $Y$ be any topological space. A Khalimsky path in $Y$ is a continuous image of a Khalimsky interval. A Khalimsky arc is a homeomorphic image of a Khalimsky interval. A Khalimsky Jordan curve in $Y$ is a homeomorphic image of a Khalimsky circle.

Sometimes Khalimsky Jordan curves are too narrow. We impose a condition on them to make their interior fatter:

Definition 2. Let $J$ be a Khalimsky Jordan curve in a topological space $Y$. We shall say that $J$ is strict if every point in $J$ is second adjacent to exactly two points in $J$.

We note that if $x, z \in J$ are second adjacent, then the intermediary $y$ required by the definition need not belong to $J$. Thus the concept of strict Jordan curve is not intrinsic.

A three-set $\{x, y, z\}$ such that all three points are adjacent to each other can be a Khalimsky path but never a Khalimsky arc. This follows from the fact that in a Khalimsky interval $[a, b]$, the endpoints $a$ and $b$ are not adjacent unless $b=a+1$. Let us say that a three-set $\{x, y, z\}$ in a topological space is a forbidden triangle if all points are adjacent to each other. The absence of forbidden triangles is therefore a necessary condition for Khalimsky arcs and consequently for Khalimsky Jordan curves, and it is often easy to check.

Different topologies may induce the same adjacency structure. However, when the adjacency structure is that of a Khalimsky circle, the topology of the space 
must also be that of a Khalimsky circle. More precisely we have the following result.

Theorem 1. Given a subset $J$ of a topological space $Y$, the following conditions are equivalent.

(A) $J$ is a Khalimsky Jordan curve.

(B) $J$ has at least four points, and for every $a \in J, J \backslash\{a\}$ is homeomorphic to a Khalimsky interval.

(C) $J$ is finite, connected, with cardinality at least 4, and each of its elements has exactly two adjacent points.

(D) $J$ has the adjacency structure of a Khalimsky circle, i.e., $J=\left\{x_{1}, x_{2}, \ldots, x_{m}\right\}$ for some even integer $m \geqslant 4$ and for each $j=1, \ldots, m, x_{j-1}$ and $x_{j+1}$ and no other points are adjacent to $x_{j}$. (Here we count indices modulo $\mathrm{m}$.)

Proof. If (A) holds, then for every $a \in J, J \backslash\{a\}$ is homeomorphic to a Khalimsky circle minus one point, thus to a Khalimsky interval. Conversely, suppose that (B) holds and consider $J \backslash\{a\}$ and $J \backslash\{b\}$ for two points $a, b \in J$ which are not adjacent. Then we have homeomorphisms of $J \backslash\{a\}$ and $J \backslash\{b\}$ into a Khalimsky circle $\mathbf{Z}_{m}$. We can modify them by rotating the circle so that the two mappings agree on $J \backslash\{a, b\}$. Then they define a local homeomorphism of $J$ onto $\mathbf{Z}_{m}$, thus a homeomorphism; we have proved (A).

It is clear that (A) implies (C) and (D).

Suppose that (C) holds. Then call an arbitrary point $x_{1}$ and one of its adjacent points $x_{2}$ and then go on, always choosing $x_{j+1}$ after $x_{j}$ so that $x_{j+1}$ is adjacent to $x_{j}$ but not equal to any of the already chosen $x_{1}, \ldots, x_{j-1}$. After a while we must arrive at a situation where there are no new points left, i.e., we arrive at $x_{m}$ and the two points adjacent to $x_{m}$ are $x_{m-1}$ and a point which has already been chosen, say $x_{k}$. A priori $k$ may be any of $1,2, \ldots, m-2$, but in fact the only possibility is $k=1$-any other choice would mean that $x_{k}$ had three adjacent points contrary to the assumption. It remains to be seen that $m$ is even. That $x_{j}$ and $x_{j+1}$ are adjacent means that we have either $x_{j} \in N\left(x_{j+1}\right)$ or $x_{j+1} \in N\left(x_{j}\right)$. If $x_{j} \in N\left(x_{j+1}\right)$, then we cannot have $x_{j+1} \in N\left(x_{j+2}\right)$, for that would imply that $x_{j}$ belonged to $N\left(x_{j+2}\right)$, so that $x_{j+2}$ would have three adjacent elements, viz. $x_{j}, x_{j+1}$ and $x_{j+3}$. So the statement $x_{j} \in N\left(x_{j+1}\right)$ holds only for $j$ of a certain parity. Since this is true modulo $m$, that number must be even. Thus we have proved (D). Conversely, (D) obviously implies (C) since (D) is just a more detailed version of $(\mathrm{C})$.

It remains to be seen that (D) implies (A). First of all it is clear that, assuming (D), $N(x)$ can never have more than three elements - a fourth element would mean that $x$ had at least three adjacent points. So $N\left(x_{j}\right) \subset\left\{x_{j-1}, x_{j}, x_{j+1}\right\}$. Considering the three points $x_{j-1}, x_{j}, x_{j+1}$, we note that either $x_{j-1} \in N\left(x_{j}\right)$ or $x_{j} \in N\left(x_{j-1}\right)$, and that $x_{j} \in N\left(x_{j+1}\right)$ or $x_{j+1} \in N\left(x_{j}\right)$. However, these alternatives cannot be chosen at will, for as we have seen in the previous paragraph $x_{j-1} \in N\left(x_{j}\right)$ implies $x_{j} \notin N\left(x_{j+1}\right)$. Consider now the case $x_{j-1} \in N\left(x_{j}\right)$. Then $x_{j+1} \in N\left(x_{j}\right)$, so that $N\left(x_{j}\right) \supset\left\{x_{j-1}, x_{j}, x_{j+1}\right\}$. On the other hand we know already that $N\left(x_{j}\right)$ has at most three elements; we conclude that $N\left(x_{j}\right)=$ 
$\left\{x_{j-1}, x_{j}, x_{j+1}\right\}$. By the same argument, $N\left(x_{j+2}\right)=\left\{x_{j+1}, x_{j+2}, x_{j+3}\right\}$. Therefore $N\left(x_{j+1}\right)=\left\{x_{j+1}\right\}$, and we have proved that $Y$ is a Khalimsky circle where points with indices of the same parity as $j$ have three-neighborhoods and points with indices of the other parity are open. The other possibility, viz. that $x_{j} \in N\left(x_{j-1}\right)$, can be reduced to the former by just shifting the indices one step.

It follows from property (C) that two Khalimsky Jordan curves can never be contained in each other. More precisely, if $J$ and $K$ are Khalimsky Jordan curves and $J \subset K$, then $J=K$.

A point on a Khalimsky Jordan curve $J$ consisting of at least six points has at least two second adjacent points; with the order introduced in property (D), $x_{j-2}$ and $x_{j+2}$ are second adjacent to $x_{j}$ and $x_{j-2} \neq x_{j+2}$ when $m>4$. Then $x_{j \pm 1}$ serve as intermediaries, but there may also exist other intermediaries. When a Jordan curve is not strict and $m>4$, then some point, say $x_{j}$, has at least one second adjacent point in addition to $x_{j-2}$ and $x_{j+2}$, say $x_{k}$. Then an intermediary $b$ such that $x_{j}$ and $b$ are adjacent and $b$ and $x_{k}$ are adjacent cannot belong to $J$.

Suppose now that $Y$ is a metric space with metric $d$. Since every Khalimsky $\operatorname{arc} \Gamma$ is homeomorphic either to $[0, m-1] \cap \mathbf{Z}$ or to $[1, m] \cap \mathbf{Z}$ for some $m$, it can be indexed as $\left\{x_{1}, \ldots, x_{m}\right\}$, where the indices are uniquely determined except for inversion. We may define its length as

$$
\operatorname{length}(\Gamma)=\sum_{1}^{m-1} d\left(x_{j+1}, x_{j}\right) .
$$

Similarly, a Khalimsky Jordan curve can be indexed as $\left\{x_{1}, \ldots, x_{m}\right\}$, where the indices are uniquely determined up to inversion and circular permutations, and its length can be defined as

$$
\operatorname{length}(J)=\sum_{1}^{m} d\left(x_{j+1}, x_{j}\right),
$$

where we count the indices modulo $m$.

We shall use the following norms in $\mathbf{R}^{2}$ to measure distances in $\mathbf{Z}^{2}$ :

$$
\|x\|_{p}=\left\|\left(x_{1}, x_{2}\right)\right\|_{p}=\left\{\begin{array}{lll}
\left(\left|x_{1}\right|^{p}+\left|x_{2}\right|^{p}\right)^{1 / p}, & x \in \mathbf{R}^{2}, & 1 \leqslant p<+\infty \\
\max \left(\left|x_{1}\right|,\left|x_{2}\right|\right), & x \in \mathbf{R}^{2}, & p=\infty
\end{array}\right.
$$

\section{Khalimsky's Digital Jordan Curve Theorem}

The Khalimsky topology of the digital plane is the Cartesian product topology of two copies of the Khalimsky line $\mathbf{Z}$. A point $x=\left(x_{1}, x_{2}\right)$ in the product $\mathbf{Z}^{2}=\mathbf{Z} \times \mathbf{Z}$ is closed if and only if both $x_{1}$ and $x_{2}$ are closed, thus if and only if both $x_{1}$ and $x_{2}$ are even; similarly $x$ is open if and only if both coordinates are 
odd. These points are called pure; the other points, which are neither open nor closed, are called mixed.

Perhaps the quickest way to describe Khalimsky's topology $\tau_{\infty}$ on $\mathbf{Z}^{2}$ is this: We first declare the nine-set

$$
U_{\infty}=\left\{x \in \mathbf{Z}^{2} ;\|x\|_{\infty} \leqslant 1\right\}=\{(0,0), \pm(1,0), \pm(1,1), \pm(0,1), \pm(-1,1)\}
$$

to be open, as well as all translates $U_{\infty}+c$ with $c_{1}, c_{2} \in 2 \mathbf{Z}$. Then all intersections of such translates are open, as well as all unions of the sets so obtained. As a consequence, $\{(1,-1),(1,0),(1,1)\}$, the intersection of $U_{\infty}$ and $U_{\infty}+(2,0)$, and $\{(1,1)\}$, the intersection of $U_{\infty}$ and $U_{\infty}+(2,2)$, are open sets, and $\{(0,0)\}$ is a closed set. The sets $\{(1,0)\}$ and $\{(0,1)\}$ are neither open nor closed.

Theorem 2. Given a subset $J$ of $\mathbf{Z}^{2}$ equipped with the Khalimsky topology, the conditions $A, B, C$ and $D$ of Theorem 1 are all equivalent to the following. (E) $J=\left\{x^{(1)}, x^{(2)}, \ldots, x^{(m)}\right\}$ for some even integer $m \geqslant 4$ and for all $j, x^{(j-1)}$ and $x^{(j+1)}$ and no other points are adjacent to $x^{(j)}$; moreover each path consisting of three consecutive points $\left\{x^{(j-1)}, x^{(j)}, x^{(j+1)}\right\}$ turns at $x^{(j)}$ by $45^{\circ}$ or $90^{\circ}$ or not at all if $x^{(j)}$ is a pure point, and goes straight ahead if $x^{(j)}$ is mixed.

Here we use the informal expression "turn by $45^{\circ}$ " etc. with reference to angles in the Euclidean plane of which we consider the Khalimsky plane to be a subset.

Proof. If (D) holds, we see that $J$ cannot turn at a mixed point and cannot turn $135^{\circ}$ at a pure point - otherwise we would have a forbidden triangle. So (D) implies (E). Conversely, (E) is just a more precise version of (D), so (E) implies (D).

In this section we shall measure the lengths of Khalimsky Jordan curves using the Euclidean metric, $d(x, y)=\|x-y\|_{2}$. It is not possible to use $\|\cdot\|_{1}$ or $\|\cdot\|_{\infty}$ in the proof of the Jordan curve theorem.

The smallest possible Jordan curve in $\mathbf{Z}^{2}$ is the four-set

$$
J_{4}=\left\{x \in \mathbf{Z}^{2} ;\|x-(1,0)\|_{1}=1\right\}=\{(0,0),(1,-1),(2,0),(1,1)\} .
$$

We add all translates of $J_{4}$ by a vector $c \in \mathbf{Z}^{2}$ with $c_{1}+c_{2}$ even and call these the Jordan curves of type $J_{4}$.

There is also a Jordan curve having eight points,

$$
J_{8}=\left\{x \in \mathbf{Z}^{2} ;\|x\|_{\infty}=1\right\}=U_{\infty} \backslash\{(0,0)\} .
$$

This curve and all its translates by a vector $c \in \mathbf{Z}^{2}$ with $c_{1}+c_{2}$ even we call the Jordan curves of type $J_{8}$.

Let us agree to call the three-set

$$
T=\{(1,1),(0,0),(1,-1)\}
$$

and rotations of $T$ by $90^{\circ}, 180^{\circ}$ and $270^{\circ}$, as well as all translates of these sets by vectors $c \in \mathbf{Z}^{2}$ with $c_{1}+c_{2}$ even, a removable triangle. It turns out that elimination of removable triangles is a convenient way to reduce Jordan curves, as shown by the following lemma. 
Lemma 1. Let $J$ be a Jordan curve in the Khalimsky plane and assume that $J$ contains the three-set $T$ defined by (4). Define

$$
J^{\prime}=(J \backslash\{(0,0)\}) \cup\{(1,0)\} .
$$

Then either $J=J_{4}$ or else $J^{\prime}$ is a Jordan curve such that $\complement J^{\prime}$ and $\complement J$ have the same number of components, and length $\left(J^{\prime}\right)=\operatorname{length}(J)-2 \sqrt{2}+2$.

Proof. Assume first that $(2,0) \in J$, thus that $J \supset J_{4}$. Then necessarily $J=J_{4}$.

Next we suppose that $(2,0) \notin J$. Then $J^{\prime}$ is a Jordan curve: $J^{\prime}$ is a set where the new point $(1,0)$ plays exactly the same role topologically as the old point $(0,0)$ in $J$. Thus $J^{\prime}$ is also homeomorphic to a Khalimsky circle.

Finally we must check that the number of components in $\complement J^{\prime}$ is the same as that of $\complement J^{\prime}$. Indeed, $(1,0)$ and $(2,0)$ belong to the same component of $\complement J$, and $(0,0)$ and $(-1,0)$ belong to the same component of $\complement J^{\prime}$.

Theorem 3 (Khalimsky's Jordan curve theorem). Let us equip the digital plane $\mathbf{Z}^{2}$ with the Khalimsky topology $\tau_{\infty}$ (see (1)). Then for any Khalimsky Jordan curve $J$ in $\mathbf{Z}^{2}$, the complement $\complement J=\mathbf{Z}^{2} \backslash J$ has exactly two connectivity components.

Proof. The complement of $J_{4}$ consists of $A=\{(1,0)\}$ and the set $B$ of all points $x$ with $\left|x_{1}-1\right|+\left|x_{2}\right|>1$. It is obvious that these two sets are connected. Moreover, $A$ is closed and open in $\complement J_{4}$, so it is a component. Therefore, also $B$ is closed and open in $\complement J_{4}$ and also a component. The proof for $J_{8}$ is similar.

Thus we know that the conclusion of the theorem holds for Jordan curves of types $J_{4}$ and $J_{8}$.

Next we shall prove that if $J$ is not of the kind already treated, then there exists a Jordan curve $J^{\prime}$ of strictly smaller Euclidean length such that $C J$ and $\complement J^{\prime}$ have the same number of components. After a finite number of steps we must arrive at a situation where the hypothesis is no longer satisfied, which means that we have a Jordan curve of type $J_{4}$ or $J_{8}$, for which the complement has two components as we already proved.

The construction of $J^{\prime}$ is as follows. First we may assume, in view of Lemma 1, that $J$ contains no removable triangles. Define

$$
a_{2}=\inf \left(x_{2} ; x \in J\right) .
$$

Thus $x_{2} \geqslant a_{2}$ for all points $x \in J$ with equality for at least one $x$. Consider a horizontal interval

$$
H=\left\{\left(x_{1}, a_{2}\right)\right\}+\{(0,0),(1,0), \ldots,(p, 0)\}
$$

which is maximal with respect to inclusion and consists of points in $J$ with ordinate equal to $a_{2}$. The maximality implies that the two points $\left(x_{1}-1, a_{2}\right)$ and $\left(x_{1}+p+1, a_{2}\right)$ do not belong to $J$. Then we see that $p$ must be an even number, but we cannot have $p=0$, since that would imply that $J$ contained a removable triangle, contrary to the assumption. Thus $H$ contains at least three 
points. Moreover, at the endpoints of $H, J$ must turn upwards. Indeed, since $\left(x_{1}-1, a_{2}\right)$ does not belong to $J$, exactly one of the points $\left(x_{1}-1, a_{2}+1\right)$, $\left(x_{1}, a_{2}+1\right)$ belongs to $J$; when we go left from $\left(x_{1}, a_{2}\right)$, the curve must turn upwards by either $45^{\circ}$ or $90^{\circ}$; it cannot turn downwards. Similarly, the curve turns upwards by $45^{\circ}$ or $90^{\circ}$ when we go right from the last point in $H$, viz. from $\left(x_{1}+p, a_{2}\right)$.

We now consider the set $\mathcal{I}$ of all maximal horizontal intervals $I$ in $J$ such that $J$ turns upwards at the endpoints of $I$. The previous argument served just to prove that there exists such an interval. Now there exists an interval $K \in \mathcal{I}$ of smallest length,

$$
K=\{y\}+\{(0,0),(1,0), \ldots,(q, 0)\}
$$

containing $q+1$ points for some even number $q \geqslant 2$. We shall assume that $K$ is of smallest length also among all intervals that can be obtained from the intervals in $\mathcal{I}$ by rotating them $90^{\circ}, 180^{\circ}$ or $270^{\circ}$.

To simplify the notation we may assume (after a translation if necessary) that $y=(0,0)$, so that

$$
K=\{(0,0),(1,0), \ldots,(q, 0)\}=[(0,0),(q, 0)] \cap \mathbf{Z}^{2} .
$$

Case 1 . $J$ turns upwards by $45^{\circ}$ at both ends of $K$. This means that $(-1,1)$ and $(q+1,1)$ both belong to $J$. In this case, we define

$$
J^{\prime}=(J \backslash K) \cup(K+(0,1)) .
$$

This operation shortens the Euclidean length by $2 \sqrt{2}-2$ (but it does not shorten the $l^{\infty}$ length). We note that the interval $K+(0,1)$ is disjoint from $J$; otherwise some point in $K$ would have three adjacent points. Moreover $K+(0,2)$ must be disjoint from $J$. Indeed, if $(K+(0,2)) \cap J$ were nonempty, then either $J$ would contain a removable triangle (contrary to our assumption) or there would exist a subinterval $K^{\prime}$ of $K+(0,2)$ contained in $J$ and such that $J$ turns upwards at its endpoints; thus $K^{\prime} \in \mathcal{I}$. This subinterval must have fewer than $q+1$ points, since $(0,2)$ and $(q, 2)$ cannot belong to $J$-otherwise there would be a removable triangle in $J$. Now a shorter interval is impossible, since $K$ is by assumption an interval in $\mathcal{I}$ of shortest length. One checks that $J^{\prime}$ is a Jordan curve. Indeed, the points of $K+(0,1)$ play the same role topologically in $J^{\prime}$ as do the points of $K$ in $J$. The number of components in the complement of $J^{\prime}$ is the same as for $J$.

Case 2. $J$ turns upwards by $90^{\circ}$ at one end of $K$. Assume that $(0,1) \in J$, the case $(q, 1) \in J$ being symmetric. Then also $(0,2) \in J$. We consider the subcases 2.1 and 2.2.

Case 2.1. $(2,2) \notin J$. We cut off a corner, i.e., we remove $(0,1),(0,0),(1,0)$, and add $(1,1)$. This operation shortens the Euclidean length by $4-2 \sqrt{2}$ (but $J^{\prime}$ has the same $l^{1}$-length as $\left.J\right)$. Since $(1,1)$ and $(2,2)$ belong to the same component of $\complement J$, and $(0,1),(0,0),(1,0)$, and $(-1,0)$ belong to the same component of $\complement J^{\prime}$, the number of components in the respective complements are the same. 
Case 2.2. $(2,2) \in J$. We consider four subcases, 2.2.1.1, 2.2.1.2, 2.2.2.1 and 2.2.2.2.

Case 2.2.1.1. $(2,1) \in J,(1,2) \in J$. Then $J$ contains a Jordan curve of type $J_{8}$, more precisely $J \supset(1,1)+J_{8}$. So $J$ must be equal to that curve.

Case 2.2.1.2. $(2,1) \in J,(1,2) \notin J$. Remove the five points $(0,1),(0,0),(1,0)$, $(2,0),(2,1)$, and add $(1,2)$. Thus $J^{\prime}$ is shorter by 4 . We can check that $J^{\prime}$ has all desired properties.

Case 2.2.2.1. $(2,1) \notin J,(1,2) \in J$. Turn $90^{\circ}$ to reduce to case 2.2.1.2.

Case 2.2.2.2. $(2,1) \notin J,(1,2) \notin J$. This case cannot occur since $q$ is smallest possible. To see this, define $I^{\prime}$ as the set of all points $(2,2),(3,2), \ldots,\left(q^{\prime}, 2\right) \in J$ with $q^{\prime}$ as large as possible. If $J$ turns upwards at $\left(q^{\prime}, 2\right)$, then $I^{\prime}$ belongs to $\mathcal{I}$ with $q^{\prime}<q$, which contradicts the definition of $K$ and $q$. If on the other hand $J$ turns downwards at $\left(q^{\prime}, 2\right)$, then there exists a vertical interval consisting of three points, which becomes an interval in $\mathcal{I}$ if we turn it $90^{\circ}$, thus again contradicting the definition of $\mathcal{I}$.

\section{The Jordan Curve Theorem for Another Topology}

We define a topology $\tau_{1}$ on $\mathbf{Z}^{2}$ by first declaring the five-set

$$
U_{1}=\left\{x \in \mathbf{Z}^{2} ;\|x\|_{1} \leqslant 1\right\}=\{(0,0), \pm(1,0), \pm(0,1)\}
$$

to be open, then all translates $U+c$ with $c \in \mathbf{Z}^{2}, c_{1}+c_{2} \in 2 \mathbf{Z}$ to be open, as well as all intersections of such translates (cf. (1)). This implies that $\{(1,0)\}$ is open, and that the origin is closed. In fact, all points $x \in \mathbf{Z}^{2}$ with $x_{1}+x_{2} \in 2 \mathbf{Z}$ are closed, and all points with $x_{1}+x_{2} \notin 2 \mathbf{Z}$ are open; there are no mixed points. This topology was described by Wyse et al. [1970] and Rosenfeld [1979:624].

The four-set

$$
J_{4}^{\prime}=\{(0,0),(1,0),(1,1),(0,1)\}
$$

is a Jordan curve for $\tau_{1}$. However, it is not strict, for a point in $J_{4}^{\prime}$ has only one second adjacent point. Its complement is connected, so the Jordan curve theorem does not hold. The set $J_{8}$ defined by (3) is a Khalimsky Jordan curve and its complement has exactly two components. Also $J_{8}$ is not strict, because the point $(1,0)$ has three second adjacent points, viz. $(0,1),(0,-1)$ and $(-1,0)$.

Another example is the twelve-set

$J_{12}$

$=\{(0,0),(1,0),(2,0),(2,1),(3,1),(3,2),(3,3),(2,3),(1,3),(1,2),(0,2),(0,1)\}$.

It is a Jordan curve, not strict, and its complement has three connectivity components, viz. an infinite component and the two singleton sets $\{(1,1)\}$ and $\{(2,2)\}$.

Theorem 4. Let $\mathbf{Z}^{2}$ be equipped with the topology $\tau_{1}$ just defined (see (5)). Then the complement of every strict Jordan curve has exactly two components. 
Proof. For the proof we shall use the fact that $\mathbf{Z}^{2}$ equipped with the topology $\tau_{1}$ is homeomorphic to the subspace of all pure points in the Khalimsky plane. This fact was used also by Kong, Kopperman and Meyer [1991:915].

Let $X$ be the digital plane $\mathbf{Z}^{2}$ with the topology $\tau_{1}$, and $Y$ the Khalimsky plane $\left(\mathbf{Z}^{2}\right.$ with the topology $\left.\tau_{\infty}\right)$. Consider the mapping $\varphi: X \rightarrow Y$ defined by $\varphi(x)=\left(x_{1}-x_{2}, x_{1}+x_{2}\right)$. Its image $\varphi(X)$ is the set of all pure points in $Y$, and if we equip it with the topology induced by $Y$ it is homeomorphic to $X$. Moreover, the image of any Khalimsky Jordan curve $J$ in $X$ is a Khalimsky Jordan curve in $Y$. Therefore $Y \backslash \varphi(J)$ has exactly two components by Theorem 3. We claim that $\varphi(X) \backslash \varphi(J)$ has exactly two components. It is clear that this set has at least two components, so the problem is to prove that a component $A$ of $Y \backslash \varphi(J)$ gives rise to a connected set $A \cap \varphi(X)$, i.e., that the pure points in $A$ form a connected set.

To this end, assume that $a, a^{\prime} \in A \cap \varphi(X)$, and consider a Khalimsky arc

$$
\left\{a=a^{(0)}, a^{(1)}, \ldots, a^{(s)}=a^{\prime}\right\}
$$

contained in $Y \backslash \varphi(J)$. (Connectedness in $Y$ is the same as arcwise connectedness; cf. Khalimsky et al. [1990: Theorem 3.2].) We shall prove that this arc can be replaced by another consisting only of pure points. So assume that $a^{(j)}$ is a mixed point. Then its predecessor $a^{(j-1)}$ and its successor $a^{(j+1)}$ are both pure points. Without loss of generality, we may assume that $a^{(j-1)}=(0,0), a^{(j)}=$ $(0,1)$, and $a^{(j+1)}=(0,2)$. We may then replace $a^{(j)}$ by one of the pure points $(-1,1),(1,1)$, because both of them cannot belong to $\varphi(J)$. To see this, suppose that $(1,1),(-1,1) \in \varphi(J)$. Then $(-1,1)$ would be a second adjacent point to $(1,1)$, and this point has, by hypothesis, exactly two second adjacent points in $\varphi(J)$ (considering everything in the space $\varphi(X)$ ). However, none of them can be equal to $(-1,1)$, for the only possible intermediaries would then be $(0,0)$ and $(0,2)$, none of which belongs to $\varphi(J)$. (In a strict Jordan curve, one of the possible intermediaries to a second adjacent point must belong to the curve.) This contradiction shows that not both of $(1,1)$ and $(-1,1)$ can belong to $\varphi(J)$. Thus we may define $b=(-1,1)$ or $b=(1,1)$ so that $b \notin \varphi(J)$ and observe that

$$
\left\{a^{(0)}, \ldots, a^{(j-1)}, b, a^{(j+1)}, \ldots, a^{(s)}\right\}
$$

is a Khalimsky arc with a mixed point replaced by a pure point. After finitely many such replacements we obtain an arc connecting $a$ and $a^{\prime}$ and consisting only of pure points. This shows that $\varphi(X) \backslash \varphi(J)$ has at most as many components as $Y \backslash \varphi(J)$; therefore exactly two components, and then the same is true of $X \backslash J$.

\section{Conclusion}

We have showed that digital Jordan curves can be subject to a simple induction process: their lengths in a suitable metric form a discrete set, and if we show that a curve has a certain property when a shorter curve has the same property, 
the induction works, starting from some very short curves. Using this idea, we have presented a short proof of Khalimsky's digital Jordan curve theorem, which is valid for the Khalimsky topology on the digital plane, where each point has eight adjacent points. We have also considered a topology for the digital plane where each points has four adjacent points. For this topology the Jordan curve theorem does not hold in general, but it does for a restricted class of curves.

\section{Acknowledgment}

I am grateful to Ingela Nyström and Erik Palmgren for helpful comments on an early version of this paper.

\section{References}

1. Aleksandrov, P. S. 1937, Diskrete Räume. Mat. Sb. 2 (44), 501-519.

2. Bourbaki, Nicolas 1961, Topologie générale. Éléments de mathématique, première partie, livre III, chapitres 1 \& 2. Third edition. Paris: Hermann.

3. Halimskiur, E. D. 1970, Applications of connected ordered topological spaces in topology. Conference of Math. Departments of Povolsia.

4. 1977, Uporyadochennnye topologicheskie prostranstva. Kiev: Naukova Dumka. pp. 92.

5. Khalimsky, Efim; Kopperman, Ralph; Meyer, Paul R. 1990, Computer graphics and connected topologies on finite ordered sets. Topology Appl. 36, 1-17.

6. Kong, Yung; Kopperman, Ralph; Meyer, Paul R. 1991, A topological approach to digital topology. Amer. Math. Monthly 98, 901-917.

7. Rosenfeld, Azriel 1979, Digital topology. Amer. Math. Monthly 86, 621-630.

8. Wyse, Frank, et al. 1970, Solution to problem 5712. Amer. Math. Monthly 77, 1119. 